Published in final edited form as:

Shock. 2017 February ; 47(2): 125-127. doi:10.1097/SHK.0000000000000781.

\title{
WHAT'S NEW IN SHOCK, FEBRUARY 2017?
}

\author{
Christopher Auger and Marc G. Jeschke \\ Ross Tilley Burn Centre, Sunnybrook Health Sciences Centre, Toronto, Ontario, M4N 3M5, \\ Canada
}

This edition of shock features a breadth of outstanding manuscripts encompassing both clinical and basic science reports. The lead-off for the former is an article by Colbert et al. (1) seeking to characterize and compare severe sepsis outcomes such as acute kidney injury (AKI), acute lung injury (ALI) and mortality in a subgroup of patients with the comorbidities of inflammatory bowel disease. Interestingly, the authors found that, counter to their hypothesis; hospitalizations with Crohn disease were associated with better sepsisrelated outcomes compared to controls. However, patients with ulcerative colitis had higher mortality and septic organ injury, illustrating the need for further investigation into the pathophysiology of these diseases.

Remaining on the topic of sepsis, Yang et al. (2) describe for the first time the procoagulant activity of neutrophil extracellular traps (NETs) in patients with sepsis. As NETs can contribute to thrombosis, the attenuation of these biomolecules is hypothesized to remediate the hypercoagulability of septic patients. Here, the authors show that that systemic inflammation stimulates neutrophils to release NETs, the reduction of which can be achieved with early anticoagulation therapy. A better understanding of NETs and their dynamics may lead to improved coagulopathy of septic patients.

One major cause of morbidity and mortality in neonates is early onset sepsis (EOS) diagnosed less than 72 hours after birth. He et al. (3) use multiplex cytokine profiling to identify IL-27 as a novel biomarker for predicting EOS when combined with procalcitonin levels. As the clinical signs for sepsis in neonates are subtle and non-specific, predictive measures for EOS are desperately needed, particularly in developing countries where the overall incidence of EOS is particularly high. The diagnostic value of IL-27 presented here can be of benefit to those hoping to lower the rate of EOS development via early intervention.

Next, Green et al. (4) study the underappreciated role of the $3^{\prime}$ untranslated region (UTR) of the human glucocorticoid receptor (hGR) which, along with glucocorticoids themselves, plays a major role in homeostasis. Interestingly, they identify a novel splice variant, hGR-S1, which lacks transactivation, DNA binding and ligand binding domains yet has a hyperactive response to exogenous steroids. They go on to further hypothesize that this variant may be produced during major stress to increase the sensitivity to endogenous corticosteroids and that variations in the hGR between patients may underlie their differential sensitivity to glucocorticoids following severe disorders such as sepsis. This research helps us understand how patients can have markedly different responses to glucocorticoids at similar doses. 
After cardiac surgery involving cardiopulmonary bypass (CPB), the susceptibility to infection increases, but there is a lack of biomarkers for the diagnosis of these postoperative complications. The work performed here by Djebara et al. (5) shows us a time course of CD64 expression and proposes the use of this glycoprotein as a marker which can discriminate between inflammation and infection following surgery. Indeed, the CD64 index was significantly higher in septic patients than in patients who had CPB, and in combination with $\mathrm{C}$ - reactive protein, it appears possible to determine infection in this population. Early diagnosis of infection can help prevent further complications such as sepsis through rapid intervention.

Remaining on the prospect of differentiating between inflammatory and infectious biomarkers, Parenica et al. (6) study the systemic response in cardiogenic shock (CS) patients. While no significant difference was found in inflammatory mediators between CS patients with or without infection, the authors describe a strong relationship between the elevation of these biomolecules and the 3-month mortality. The hope is that these results may lay the groundwork for a prospective study to evaluate the combination of inflammatory markers in CS patients and better inform the initiation of antibiotic therapy in cases of suspected infection.

In the final clinical report, Holmes et al. (7) analyzed the cholinergic mediators in both donor and burn margin skin from human burn patients and compared these to skin from unburned controls. While skin grafts accelerate the healing of burn wounds, autologous grafts may display large-scale deterioration and/or functional deficits which lead to graft failure. To understand this aberrant response, the authors elect to explore the epidermal cholinergic pathway, which is known to modulate the local inflammatory response and barrier permeability function. They found that a decrease in CHRNA7 accompanies an increase in SLURP1 and the ratio of ACh/ACHE, implicating cholinergic mediators in burn injury. The downstream increase in HMGB1 and caspase 3 indicate the activation of apoptotic and necrotic pathways. As such, cholinergic agonists/antagonists may modulate tissue apoptosis and necrosis to improve the outcomes of skin grafts in burn patients.

In the category of basic science, Klingensmith et al. (8) look at the comorbidity of alcohol use in septic mice to determine if chronic alcohol ingestion impedes the cytoprotective effects of epidermal growth factor (EGF). One of the predominant locations where EGF exerts its beneficial effects is the intestinal epithelium, whose integrity is worsened by alcohol consumption. Here, intestinal permeability was assessed by monitoring the tight junction proteins claudin 5 and JAM-A as well as apoptosis via Bcl-2 and Bax. The authors show that EGF improves survival in both alcohol- and water-fed mice, but that the efficacy of this mediator is blunted in the alcohol cohort, further establishing the detrimental effects of chronic alcohol consumption on health outcomes.

Hafner et al. (9) describe the role of the purinergic receptor P2XR4 in mice after blunt trauma in mice exposed to cigarette smoke (CS). As both P2XR4 and P2XR7 is significantly up-regulated following trauma, their role in the acute pulmonary inflammatory response merits further investigation. Here, the authors show that the deletion of P2XR4 had no effects on pulmonary function and inflammatory response, and neither did short term CS 
exposure. However, deletion of the purinergic receptor was associated with attenuated impairment of glucose homeostasis. This work furthers our understanding of the signaling pathways underlying pulmonary hyperinflammation.

In keeping with pulmonary injuries but shifting the context to burns, Liu et al. (10) evaluate the applicability of a closed-loop control of $\mathrm{FiO}_{2}$ algorithm to rapidly assess acute respiratory distress syndrome (ARDS) severity in a sheep model of inhalation injury. The benefits of these smart oxygenation systems for combat casualties and burn patients include the rapid recognition of pulmonary injury severity and the earlier initiation of rescue ventilation (RscVent). In the conscious sheep model, this life saving intervention lead to improved oxygenation and ventilation outcome. The technology has exciting translatability for cases of human trauma.

Next, Chen et al. (11) use a rat model of polytrauma and hemorrhage to demonstrate the utility of whole blood in restoring metabolic and hemostatic function after severe injury. Similarly to human blood stored at $4^{\circ} \mathrm{C}$, rat blood kept for 10 days exhibits a prolongation in prothrombin time (PT) and partial thromboplastin time (aPTT) and fall in fibrinogen concentration. Use of this blood, either fresh or stored, returned arterial pressure, plasma lactate and plasma bicarbonate near control levels in the injured animals. However, no corrections to PT, aPTT or platelet dysfunction were observed, outlining the need for more clotting factors than provided by the whole blood alone. Knowledge of the best means by which to store blood and the additives required to ameliorate its function may improve the outcomes of blood transfusions.

In keeping with blood, Zettel et al. (12) demonstrate how the transfusion of red blood cells (RBCs) can be a double-edged sword, being a necessary part of resuscitation for patients but also inducing a state of relative immunosuppression. They propose that elements of RBCs can act as damage-associated molecular patterns (DAMPs) which impair microbial clearance and identify one such mediator, HMGB1, which is released from RBC units during storage. The authors show that this particular DAMP can impair the clearance of Escherichia coli in a two-hit trauma/infection model and postulate that removal of HMGB1 from RBCs can improve transfusion results. This is interesting research, as we're accustomed to adding components to improve blood, yet here we see that certain endogenous compounds may need to be eliminated for more successful transfusions.

Bruns et al. (13) show in their elegantly-conducted study that neutrophils are a necessary component of Fas-induced lung injury in neutrophil-reduced mice. Pulmonary Fas activation is an important step in the pathogenesis of ALI and ARDS, but it was unknown if it relied on the migration of activated PMNs into the lung. The authors demonstrate that while apoptosis is independent of PMN, for full Fas-induced lung injury to develop, neutrophil recruitment is a mandatory component. This work further elucidates the signaling underlying lung injuries.

Returning to CS, Vanhuyse et al. (14) demonstrate the benefits of rapidly-induced moderate hypothermia in a pig model of ischemic CS. Briefly, the authors conclude that this drop in temperature improves hemodynamics, cardiac and vascular function in their model with the added benefits of being simple and inexpensive to implement in clinic. Optimization of 
veno-arterial extracorporeal membrane oxygenation with simple strategies such as the induction of hypothermia may lower the mortality rates of CS in patients.

Fujioka et al. (15) use a cecal slurry model in 4 day-old mice to mimic the septic condition of human neonates and study the role of heme oxygenase-1 (HO-1). The latter is thought to exert a protective role via its anti-inflammatory, antioxidative and anti-apoptotic functions. To confirm this, heme was administered to mice prior to the induction of sepsis and the increased activity of HO-1 was observed in the liver and spleen, which was associated with decreases in the development of sepsis and incidence of mortality. Understanding how HO-1 confers protection against infant sepsis may lead to improved strategies to regulate this enzyme.

The final basic science report comes from Ge et al. (16) and discusses the increase in stromal cell-derived factor-1 (SDF-1) and its receptor CXCR4 in side population cells during renal ischemia-reperfusion (I/R) injury. The authors demonstrate how this signaling is protective and may account for kidney repair after I/R injury via the Shh-Gili1 pathway. As $\mathrm{AKI}$ is associated with high morbidity and mortality following severe injury, a greater understanding of the repair pathways may lead to novel therapeutic strategies to improve on this outcome.

To that end, we hope you thoroughly enjoy this fantastic issue of shock, highlighting some of the impressive advances in research aimed at improving trauma outcomes and elaborating on the mechanisms underlying infections, sepsis and pulmonary disorders.

\section{References}

1. Colbert JF, Schmidt EP, Faubel S, Ginde AA. Severe sepsis outcomes amonghospitalizations with inflammatory bowel disease. Shock. 2017; 47:000-000.

2. Yang S, Qi H, Kan K, Quan C, Xie H, Guo X, Zhang L. Neutrophilextracellular traps promote hypercoagulability in patients with sepsis. Shock. 2017; 47:000-000. SHOCK MONTH 2016 FEBRUARY 2017 COMMENTARY 3.

3. He Y, Du WX, Jiang HY, Ai Q, Feng J, Liu Z, Yu JL. Multiplex cytokineprofiling identifies interleukin-27 as a novel biomarker for neonatal early onset sepsis. Shock. 2017; 47:000-000.

4. Green TL, Leventhal SM, Lim D, Cho K, Greenhalgh DG. A tri-nucleotidepattern in a 30 UTR segment affects the activity of a human glucocorticoid receptor isoform. Shock. 2017; 47:000-000.

5. Djebara S, Biston P, Fosse' E, Daper A, Joris M, Boudjeltia KZ, Lelubre C, Cauchie P, Piagnerelli $\mathrm{M}$. Time course of CD64, a leukocyte activation marker, during cardiopulmonary bypass surgery. Shock. 2017; 47:000-000.

6. Parenica J, Jarkovsky J, Malaska J, Mebazaa A, Gottwaldova J, Helanova K, Litzman J, Dastych M, Tomandl J, Spinar J, et al. On behalf of the GREAT Network. Infectious complications and immune/ inflammatory response in cardiogenic shock patients: a prospective observational study. Shock. 2017; 47:000-000.

7. Holmes CJ, Plichta JK, Gamelli RL, Radek KA. Burn injury alters epidermalcholinergic mediators and increases HMGB1 and Caspase 3 in autologous donor skin and burn margin. Shock. 2017; 47:000-000.

8. Klingensmith NJ, Yoseph BP, Liang Z, Lyons JD, Burd EM, Margoles LM, Koval M, Ford ML, Coopersmith CM. Epidermal growth factor improves intestinal integrity and survival in murine sepsis following chronic alcohol ingestion. Shock. 2017; 47:000-000. 
9. Hafner S, Wagner K, Weber S, Gröger M, Wepler M, McCook O, Scheuerle A, Stahl B, Huber-Lang M, Jung B, et al. RoleofthepurinergicreceptorP2XR4after blunt chest trauma in cigarette smokeexposed mice. Shock. 2017; 47:000-000.

10. Liu NT, Salter MG, Khan MN, Branson RD, Enkhbaatar P, Kramer GC, Salinas J, Marques NR, Kinsky MP. Closed-loop control of FIO2 rapidly identifies need for rescue ventilation and reduces ARDS severity in a conscious sheep model of burn and smoke inhalation injury. Shock. 2017; 47:000-000.

11. Chen J, Wu X, Keesee J, Liu B, Darlington DN, Cap AP. Limited resuscitationwith fresh or stored whole blood corrects cardiovascular and metabolic function in a rat model of polytrauma and hemorrhage. Shock. 2017; 47:000-000.

12. Zettel KR, Dyer M, Raval JS, Wu X, Klune JR, Gutierrez A, Triulzi DJ, Billiar TR, Neal MD. Aged human stored red blood cell supernatant inhibits macrophage phagocytosis in an HMGB1 dependent manner after trauma in a murine model. Shock. 2017; 47:000-000.

13. Bruns B, Hönle T, Kellermann P, Ayala A, Perl M. Divergetn effects of neutrophils on Fas-induced pulmonary inflammation, apoptosis, and lung damage. Shock. 2017; 47:000-000.

14. Vanhuyse F, Ducrocq N, Louis H, Al Kattani N, Laurent N, Joineau-Groubatch F, Falanga A, Maureira J-P, Kimmoun A, Girerd N, et al. Moderate hypothermia improves cardiac and vascular function in a pig model of ischemic cardiogenic shock treated with veno-arterial ECMO. Shock. 2017; 47:000-000.

15. Fujioka K, Kalish F, Zhao H, Lu S, Wong S, Wong RJ, Stevenson DK. Inductionof heme oxygenase-1 attenuates the severity of sepsis in a non-surgical preterm mouse model. Shock. 2017; 47:000-000.

16. Ge G, Zhang H, Li R, Liu H. The function of SDF-1-CXCR4 axis in SP cellsmediated protective role for renal ischemia/reperfusion injury by SHH/GLI1ABCG2 pathway. Shock. 2017; 47:000 000 . 